Pour la version publiée, voir : Régimbeau Gérard, 2001. Clés d'accès aux images fixes : indexation et perspectives pédagogiques à partir des ressources d'Internet, Spirale (Lille 3), $\mathrm{n}^{\circ}$ 28, p. 233-251. Disponible en ligne, URL : http://spirale-edu-revue.fr/spip.php?article955

Gérard RÉGIMBEAU

\title{
CLÉS D'ACCÈS AUX IMAGES FIXES : INDEXATION ET PERSPECTIVES PÉDAGOGIQUES À PARTIR DES RESSOURCES D'INTERNET
}

Résumé : Internet permet d'interroger des banques d'images représentatives de divers types de collections et systèmes de traitement documentaire. On peut inclure maintenant leur étude et leur utilisation dans le cadre d'enseignements qui souhaiteraient jeter des ponts entre techniques documentaires et pédagogie de l'image. Chaque site présenté dans l'article fait l'objet d'un commentaire où sont proposées quelques problématiques en vue de construire des exercices sur l'analyse, l'indexation et la description, puis, des réflexions générales rappellent en quoi l'optique documentaire peut aider l'élève dans une approche critique des images.

Mots-clés : Internet. Banque d'images numérisées. Pédagogie de l'image. Pédagogie de la documentation. Indexation de l'image. Analyse documentaire de l'image. Sémantique de l'image. Description. Connotation. Recherche de l'image par l'image.

\footnotetext{
Abstract : Access keys to Still Images : Using Internet resources for Indexing and Teaching Purposes.

On the Internet, one can access and search image banks featuring different types of_collections and different types of information processing systems. It is now possible to include them in courses aiming at building bridges between information processing techniques and the study of still images. Every site featured in this article is first commented, some theoretical principles are suggested with a view to devising exercises about the analysis, description and indexing of still images ; then general observations are made about how an information processing perspective can help students deal with the critical analysis of still images.
}

Keywords :

Internet. Digital Image Bank. Image Teaching. Information Processing Teaching. Documentary content analysis of still images. Image indexing. 
Image semantics. Description. Connotation. Image search by visual content.

\section{Introduction}

On ne sait pas encore si l'on peut apprendre à voir, comme en témoignait le titre interrogatif d'un colloque relativement récent ${ }^{1}$. Malgré les nombreuses tentatives déployées dans les écoles, les lieux de culture et de formation les plus divers, malgré l'effort de vulgarisation de certains auteurs, qu'ils soient critiques, journalistes, historiens, conservateurs, artistes ou réalisateurs, la question de l'éducation à l'image reste encore ouverte. On invoque souvent un manque de mobilisation autour de ses enjeux, mais il faut aussi concevoir, plus simplement, que cette question déplace avec elle les réponses qu'on pourrait lui apporter selon un renouvellement constant. Chaque génération se trouve ainsi confrontée à des données nouvelles qui demandent une adaptation méthodologique de la part des pédagogues et des chercheurs. La prise en compte d'Internet parmi les canaux et supports multiples de diffusion de l'image fixe s'impose depuis quelques années, suivant en cela l'augmentation de son impact social, comme une des adaptations nécessaires.

Si ce contexte actuel de diffusion massive, grâce aux techniques numériques, soulève des difficultés et des interrogations d'ordre technique, économique, éthique ou esthétique - on connaît, entre autres, les problèmes relatifs à la concentration des agences, aux droits d'auteurs ou aux conséquences formelles du numérique ${ }^{2}$ - il offre aussi des atouts dont on peut tirer parti dans une perspective comparatiste et critique.

Le tranfert et la démultiplication sur Internet de services voués à l'éducation et la culture, à la promotion d'activités lucratives, associatives ou de recherche, et, directement, à la vente en ligne permettent de se confronter maintenant, à partir d'un seul vecteur, aux contrastes d'une offre qui implique, pourvu qu'on la détecte et qu'on la mette en évidence, une économie de l'image. Économie au sens marchand mais encore organisation et structuration des éléments d'un ensemble, comme on parle de l'économie d'un ouvrage. Mais, ce n'est pas le statut ou le rôle de l'image utilisée en tant qu'illustration, matériau graphique, création artistique ou document informatif qui sera ici retenu. Notre intérêt se portera à l'amont de cette utilisation, vers des sites fournisseurs d'images.

1 Peut-on apprendre à voir ? Sous la dir. de Laurent Gervereau (1999).

2 Cf. en particulier pour le photojournalisme les articles de Ange-Dominique Bouzet, Les photographes en émoi pour leurs droits, Libération, mercredi 30 août 2000, p. 26 et de Michel Guerrin, Photoreporters, les illusions perdues, Le Monde, 6 sept. 2000, p. 15. 
Dans cette optique, il devient possible, en s'appuyant sur la capacité d'Internet à réunir et déployer de larges gammes d'information, tant du point de vue de ses natures et de ses formes que de ses contenus, d'accéder aux diverses règles concurrentes, coïncidantes ou conjuguées des traitements documentaires. L'objectif de cet article sera donc d'attirer l'attention sur certaines sources iconographiques maintenant disponibles, sans prétendre sectoriser leurs utilisations pédagogiques par classe ou par niveau, ainsi que d'apporter quelques réflexions, nous l'espérons incitatives, sur les passages à explorer entre techniques documentaires et pédagogie de l'image.

\section{DES TECHNIQUES DOCUMENTAIRES POUR ABORDER L'IMAGE}

\subsection{Traiter l'information}

Les techniques documentaires n'ont pas pour seul objectif de traiter des supports. La notion même de document, telle qu'elle a été définie par Jean Meyriat (1993, p. 152) : "Un document est un objet sur lequel sont enregistrés des signes représentant de l'information", met l'accent autant sur la composante matérielle (objet dont il convient ici d'agrandir l'acception à celle de support) que sur les composantes sémiques et informationnelles qu'il revient à la documentation de traduire et de catégoriser en vue de leur exploitation.

Même si elle n'est pas ordinairement convoquée parmi les savoirs consacrés à l'interprétation du sens, la documentation a pourtant son rôle à jouer dans l'approche des contenus : une dimension clairement illustrée par les démarches d'analyse et d'indexation ${ }^{3}$. Elle ne se confond pas, à moins de l'évaluer trop superficiellement, avec un simple travail de transmission aveugle et sans effet cognitif. De plus, traitement informatique n'est pas encore synonyme d'automatisation complète ; comme on pouvait le lire en conclusion d'un article sur les moteurs de recherche d'images numérisées : "La recherche par indexation textuelle a encore de beaux jours devant elle, qu'on se le dise" (Mandard, 2000, p. III). La rédaction de légendes, descriptifs, textes d'accompagnement et la création des indexats (mots-clés, descripteurs, termes libres ou contrôlés et autres représentations chiffrées ou codifiées) relèvent avant tout d'opérations intellectuelles imposant des choix, des sélections, un lexique, une syntaxe, et en fin de compte, d'une construction de sens. Si, dans leurs résultats, ces opérations paraissent "sans qualités" puisqu'elles repoussent - jusqu'à un certain point - toute emprise ou rhétorique de la subjectivité, et répondent à une fonction intermédiaire de transmission, puisque leur objectif fondamental est de permettre la recherche par sujet, elles se concrétisent néanmoins dans un "langage" qui résulte bien de facultés de compréhension et d'intelligence d'une médiation humaine. "Sémaphorique" en ce qu'elle caractérise, balise et rappelle à l'attention

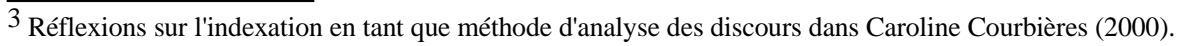


des documents par de nouveaux signes, l'indexation implique une véritable prospection de la matière informative afin d'en dresser des relevés. Lorsqu'elle est appliquée à l'image, elle présente, en outre, la particularité de désigner des unités iconiques à l'aide d'unités verbales ; ce qui nécessite la recherche de correspondances pertinentes entre les univers langagier et visuel.

\subsection{Un atout complémentaire pour la pédagogie de l'image}

Pour ces raisons brièvement évoquées, et pour d'autres qui tiennent à la mise en pratique transversale de connaissances acquises dans d'autres secteurs (expérience commune, culture personnelle, enseignements scolaires ou universitaires, disciplines scientifiques), l'analyse et l'indexation documentaires forment l'axe d'une méthode qui peut donner matière à réflexion pour une pédagogie qui sollicite les facultés d'observation, d'induction et de déduction chez l'élève. Sans prétendre remplacer des projets qui initient à la compréhension des composants et des codes visuels, tels qu'ils sont incarnés, par exemple, dans des manuels didactiques comme Petite fabrique de l'image (1992) ou Dix modèles d'analyse d'image (1995), l'approche de l'image fixe ramenée à son statut de document iconique (selon la terminologie normalisée ${ }^{4}$ ) peut s'avérer un moyen auxiliaire efficace pour structurer la perception autour d'une verbalisation particulière (la condensation ${ }^{5}$ ) et découvrir certains aspects oubliés ou masqués du circuit des images. Son avantage et son apport consistent à quitter le cadre traditionnel du commentaire de document figuré - si tant est que la régularité de son exercice en ait fait une activité traditionnelle (!) - et de déplacer l'attention du caractère "exemplaire" des documents proposés vers des territoires où le sens attribué aux images est, en quelque sorte, en état d'incubation.

Internet, pour l'ensemble des images qu'il véhicule, apparaît au premier abord comme une photothèque sans mur, mais ceci n'est qu'une métaphore incomplète ou approximative. Même s'il est possible, à partir des pages d'interrogation d'annuaires et moteurs de recherches, de retrouver des documents figurés, on n'est pas pour autant conduits vers des collections traitées et organisées pour servir de ressources 6 . Pour qui souhaite approcher les différents systèmes d'indexation des images fixes, il faut donc orienter l'interrogation vers les banques d'images, et sur ce plan, Internet constitue une vitrine assez représentative. Loin s'en faut que leurs exemples soient tous pertinents ou présentent un égal intérêt ; ils n'en restent pas moins assez significatifs des questions et méthodes en jeu dès qu'il s'agit de caractériser, de classifier et de classer des images dans un contexte documentaire.

\footnotetext{
${ }_{5}^{4}$ Documents iconiques. ISO 5127-3 (1988).

5 Réduction d'information qui peut se traduire par une notice, une légende ou des indexats.

6 Pour ce qui concerne les questions générales relatives à la recherche d'images sur Internet, cf. Anne-Marie Moulis (1999).
} 


\section{BANQUES D'IMAGES}

\subsection{Le choix des sites}

Pour une synthèse rapide et un panorama compréhensif des différents sites consultables, il existe plusieurs guides. La rubrique "Autres sites" de BIPS (Banque d'images pédagogiques et scénarios) 7 , les listes de l'ADBS (Association des professionnels de l'information et de la documentation) $^{8}$, de l'INRIA (Institut national de recherches en informatique et automatique $)^{9}$ à la rubrique "Webthèque", et le répertoire Iconos de la Documentation française ${ }^{10}$, sont des points de départ nécessaires. Ils recensent les adresses de banques d'images en ligne en donnant une sélection significative de différents domaines. A ceci, il faut ajouter les portails de la Médiathèque du Musée d'art contemporain de Montréal ${ }^{11}$, aux orientations foisonnantes, et du Ministère de la culture français ${ }^{12}$ qui regroupe les liens des banques de données consacrées aux oeuvres du patrimoine national.

Muni de ces quelques signets, il faut ensuite tester les différents sites, puis différencier les domaines thématiques et linguistiques concernés. Il ressort de ces navigations qu'une large part des banques d'images inventoriées sont accessibles, certes, mais pour un usage professionnel qui nécessite de passer par l'utilisation payante des services proposés. Soit pour des raisons commerciales, soit parce que leurs fonds ne sont pas numérisés, les agences utilisent ainsi le réseau pour faire connaître leurs activités mais sans ouvrir au-delà leurs trésors iconographiques. Pour les plus engageantes, on a accès - avec Giraudon $^{13}$, par exemple - à quelques reproductions qui reflètent l'éventail des collections mais sans autre possibilité d'interrogation que d'entrer en contact avec le service de recherches iconographiques. Quand, à ce stade, on souhaite formuler une demande, la suite relève du traitement payant d'une commande ordinaire. D'autres agences ont choisi une stratégie commerciale différente en ouvrant une partie de leurs fonds à la consultation directe.

Parmi les sites consacrés, entièrement ou en partie, aux images fixes, nous retiendrons ceux qui permettent d'observer à la fois les termes utilisés dans l'indexation et les images elles-mêmes ${ }^{14}$. Il n'est pas si fréquent de rencontrer ces deux éléments réunis, et quand ils le sont, encore faut-il séparer les systèmes évolués des systèmes rudimentaires.

Lorsqu'on interroge, par exemple, une agence spécialisée dans la vente de posters, on accède à des catalogues promotionnels, appuyés sur

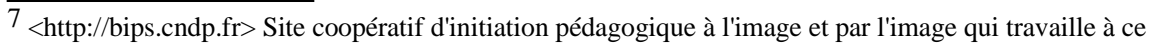
rapprochement souhaité entre matières enseignées et documentation visuelle.

$8<$ http://www.adbs.fr>

$9<$ http://www.inria.fr/multimedia/hypertheque>

$10<\mathrm{http} / /$ www.admifrance.gouv.fr/cgi-bin/multitel/CATALDOC> : voir la rubrique "Documentation", puis "Photographie" et, enfin, dans le menu de cette dernière : "Iconos" et "Banques d'images"

$11<\mathrm{http}: / /$ macm.qc.ca>

$12<$ http://www.culture.fr/culture>

13 <http://www.giraudon-photo.fr>

${ }^{14}$ La fonction "Aide" en page d'accueil des sites précise généralement les possibilités d'accès et les procédures d'interrogation.
} 
quelques pièces représentatives, arrêtant la compréhension des rapports entre image et classification à des catégories larges telles que "Mer, Montagne, Campagne", etc. Ce premier degré ne permet pas des recherches fines sur le travail documentaire, il peut tout au plus informer le "consultant apprenant" sur un niveau thématique général qui répond essentiellement à une utilité commerciale dépendante d'un support particulier, à l'instar d'un dépliant d'échantillons. Cependant, on notera déjà, pour ce cas, qu'une analyse pragmatique y découvrira les raisons d'un "classement". Il ne s'agit pas pour ces agences d'archiver des fonds d'images et d'en extraire une indexation profonde : seuls quelques critères essentiels suffisent à se repérer dans un ensemble clairement identifié.

D'autres sites offrent, en revanche, de véritables terrains d'observation des méthodes d'indexation. On en retiendra plusieurs types qui correspondent à l'image d'illustration, au photojournalisme, aux oeuvres d'art, à l'image scientifique et technique, auxquels nous ajouterons un site expérimental de recherche d'image par l'image. Pour l'ensemble de ces domaines, les pratiques sont variables et reflètent une sorte d'état de la question. Elles nous apprennent, en particulier, que les évolutions plus ou moins récentes en matière de documentation iconographique se sont effectuées selon des logiques, pour la plupart, autonomes, et - c'est là un intérêt qui n'est pas des moindres - elles permettent de constater qu'Internet, en mettant en regard et en comparaison ces divers systèmes, milite, peut-être malgré lui, pour leur mixage productif.

\subsection{Images et critères d'indexation}

La scène "primitive" d'une demande de document dans une photothèque a été brossée avec humour et vérité par J.P. Leguéré dans un article sur la documentation iconographique ${ }^{15}$ :

-"Bonjour madame! J'voudrais un lion...

-Un lion? Mais quel lion? se récrie la documentaliste de la photothèque. Mort ou vif ? D'Afrique, d'Inde ou d'Asie ? Seul ou en famille ? En gros plan ou dans son environnement ? De face ou de trois quarts ? En chasse ou au repos? Pour illustrer un poster, un livre, une annonce, un conditionnement?".

Loin de nous replonger dans des temps archaïques de la documentation manuelle, cet instantané du quotidien des services et agences iconographiques saisit ce qui constitue le trait le plus difficile et le plus sensible du travail documentaire appliqué à l'image, à savoir la recherche de l'adéquation entre les contenus de l'image, les termes de l'indexation, les mots de l'interrogation et les attentes de l'usager. Et quel que soit l'outil concerné, manuel ou informatique, cette difficulté demeure, tout au moins dans la détermination des sujets. Car, si globalement, il est assez facile de retrouver des images par auteur, titre, date, agence, éditeur, support, le critère du sujet, quant à lui, reste ouvert

$\overline{15 \text { BàT : Bon à Tirer, 1980, n }}$ 21, troisième partie, p. 62. 
à de multiples variations. Ce sont les différentes façons de résoudre cette question qu'on retrouve dans les quelques sites sélectionnés.

\subsubsection{Images d'illustration : Pictor}

Pictor, présente le cas d'une agence d'illustration dont les produits sont essentiellement destinés à la publicité et à l'édition imprimée ou numérique ${ }^{16}$. Elle possède un fonds composé de photographies et montages photographiques ouvert au "visiteur", et pratique une indexation (en anglais) par termes descriptifs ${ }^{17}$, morphologiques et connotatifs. Plusieurs entrées sont prévues à partir, entre autres, de fonctions intitulées "Category search", "Keyword search"et "Visual search".

La partie "catégorie" reprend les divisions retenues habituellement dans ce type d'agence, à savoir l'agriculture, les animaux, la finance et les affaires, la nourriture, etc. Ce premier degré d'interrogation permet de visualiser des stocks d'images importants selon un affichage mosaïque de 25 imagettes par page (ex : 1771 réponses pour la rubrique "Animals" soit 71 pages-écran consultables). La navigation, comparable au feuilletage d'un catalogue, - et qui exige de la patience avec un matériel aux capacités ordinaires - s'effectue dans ce cas par vision directe, sans mots intermédiaires si ce n'est le terme générique indexant la catégorie.

La recherche par "mot-clé" (keyword), qui concerne l'ensemble des collections, offre, quant à elle, la possibilité d'associer des termes (opérateurs booléens : "must have", "and either", "but not") qui affine la sélection d'images. Pour revenir à l'exemple du lion - on sera en mesure d'obtenir une photographie au format horizontal d'un lion dans la savane en associant successivement "lion" et "savannah", puis en repérant une photographie horizontale dans ce premier tri visualisable. Si les réponses ne convenaient pas, on pourra reprendre la requête avec les termes "lion" et "horizontal", etc.

L'illusion serait de penser que c'est le contenu effectif de l'image qui permet de la retrouver, or, si un ciel visible et manifeste dans telle photographie n'a pas été décrit par le terme "ciel" ("sky") au moment de l'indexation, on ne pourra pas accéder à cette photographie par l'intermédiaire de ce terme. La liste des indexats retenus apparaît en activant le bouton "get info". Prenant acte de ces procédés, on pourra exercer le sens critique de l'élève en lui demandant de vérifier la pertinence de termes affectés à une image prise dans un contexte, en tenant compte des impératifs d'utilisations qui conditionnent, en quantité et en qualité, le choix des termes. Il ne s'agira plus de rechercher des mots qui conviendraient pour nommer les contenus d'une image isolée pratique courante dans l'analyse descriptive de document figuré - mais de définir auparavant le cadre d'indexation : si telle image était destinée au

\footnotetext{
16 <http://www.pictor.fr>

17 Il est difficile de reprendre, ici, la terminologie du traitement documentaire de l'écrit sous peine de forger l'expression pléonasmatique "descripteurs descriptifs" (!). L'expression "descripteurs de contenu" serait plus acceptable si elle ne laissait, alors, supposer que la morphologie ne fait pas partie intégrante du contenu.
} 
domaine géographique, quels aspects faudrait-il retenir, et d'abord seraitelle exploitable dans ce domaine ? si on souhaitait qu'elle soit retrouvée par des néophytes ou au contraire par des spécialistes, quel vocabulaire serait envisageable ? etc. Entre les "mots" censés découler directement de l'image et les mots qui vont à l'image, il y a une distance que l'on suppose mais qu'il est bon, aussi, d'éprouver. Tout l'art sera de ne plus rassembler les termes selon une encombrante exhaustivité, ou plutôt volonté d'exhaustivité, mais de les distinguer et les sélectionner en fonction d'une pertinence qui demande des réglages préalables : quel destinataire viser ? quel temps accorder au travail d'indexation ? quelle originalité relever dans telle image par rapport à une série ? etc. Un de ces réglages consiste à recourir à une liste pré-organisée de termes sous les formes du vocabulaire, de la liste de vedettes matières ou du thésaurus. En passant du langage naturel, ouvert et polysémique aux langages particuliers et contrôlés qui fondent ces outils d'indexation, l'élève pourra prendre ici la mesure de la spécificité du lexique par rapport au dictionnaire et des contraintes de ses applications.

Mais, le support d'une réflexion plus ample, et très certainement plus attractive, nous est offert par la rubrique "Visual search" qui ordonne dans une liste fermée une trentaine de termes connotatifs (adventure, aggression, ambition, bizarre, calm, celebration, challenge, communicaton, etc) reliés à des groupes d'images. Dans le droit fil des études sémiologiques portant sur la publicité ${ }^{18}$ les agences d'images ont développé des systèmes d'indexation qui prennent en compte les sens latents et/ou culturels des images pour répondre aux besoins de leurs clients et anticiper les demandes en matière d'illustration. Un graphiste ou publicitaire, face au choix d'une image évocatrice ou suggestive, est confronté à ces questions. Quelle image associer, par exemple, en couverture d'un magazine, à un titre sur le stress dû au manque de temps ? En consultant la partie "concept word" (où sont rassemblées des images référées à des idées, des sentiments et des concepts) ils sélectionnera "aggression" ou "time" pour rechercher, parmi les images proposées, celle qui répondrait à ses attentes.

Tout repose ici sur des codifications rarement aussi explicites dans les expériences courantes de l'image. C'est comme si on retournait au point précis où le sens culturel investi dans les images était cristallisé ; au lieu où elles recoivent un barème, où le sens qu'on leur affecte dans nos médias occidentaux était repris et prolongé. Il suffit de tenter une interrogation sur "happiness" pour comprendre quelles images, au double sens, propre et figuré (et c'est bien ici le cas de le dire !), sont régulièrement associées à ce "concept" : couples, familles, adultes et enfants, rires et sourires, danse, jeux, etc. tout un ensemble décrivant un bien-être d'apparence, principalement décliné sur le mode familial, illustre le "propos". Les réponses pour "paradise" sont aussi significatives - ce qui n'empêche pas de goûter les variations iconiques de cette uniformité "conceptuelle" - : tropiques, mers tranquilles, plages et palmiers composent les vues idyliques de ces paradis vierges et

\footnotetext{
18 En particulier par Roland Barthes (1964, p. 40-51) dans son étude sur une publicité de Panzani.
} 
inhabités qui sont descendus des gravures et récits anciens pour saturer maintenant toute iconographie de l'évasion ${ }^{19}$.

De l'étude des réactions culturelles et subjectives en jeu dans ce type d'indexation à la recherche de termes connotatifs supplémentaires, comparés et commentés en groupe pour souligner recoupements et différences, on rejoindra certains des exercices proposés dans Petite fabrique de l'image ${ }^{20}$ avec l'avantage de disposer là des sources qui guident l'amplification connotative de ces images dans les productions publicitaires et éditoriales. En présence d'exemples aussi significatifs, notamment face aux images indexées dans la rubrique "bizarre" dont l'esthétique plus ouvertement expressionniste ou surréalisante accroche encore mieux des projections subjectives, des tests et expérimentations sur les relations, parfois intriquées, entre description et interprétation, mettront en évidence les parts personnelle et collective de la charge connotative. On sera confronté, de fait, aux questions qui assaillent les documentalistes au seuil de toute indexation connotative ${ }^{21}$.

\subsubsection{Photojournalisme : Sygma}

Le site de l'agence Sygma ${ }^{22}$ permet des recherches sur des lots d'images qui illustrent certains évènements politiques, sociaux et culturels répondant aux besoins de la presse d'actualité et de la presse dite "people". Les séries de photographies accessibles au visiteur sont regroupées par titre dans la partie "reportages". Du titre de reportage, on passe à l'affichage mosaïque des photographies puis à la consultation de chaque photographie accompagnée de sa "fiche technique" et des "motsclés"23.

Ces derniers consistent en une liste de descripteurs, parfois nombreux, qui mêlent niveaux généraux et spécifiques relatifs à l'image affichée. Ainsi tel cliché d'une réunion de personnalités du monde politique sera "relayé" par des termes qui concernent la date, le lieu, les noms des personnes, leurs fonctions, le domaine (ex. : France politique), les motifs de la réunion, etc. ; par des termes relatifs à la morphologie de l'image (ex : photo numérique, vue rapprochée), ainsi que par des termes décrivant gestes et composants (ex. : lisant, debout, table). Une fonction "Rebondir" est prévue qui permet, après avoir coché certains des "motsclés", d'obtenir l'affichage d'autres photographies indexées par des mots identiques.

Ce type d'indexation, adapté aux besoins de la presse, se prête à une analyse des différents termes retenus en séparant, d'une part, ceux

\footnotetext{
19 Cf. également notre chapitre "Signe plastique et signifiés" dans G. Régimbeau (1996, p. 289-298).

20 Notamment les exercices 10 "Le bestiaire iconographique et littéraire", 11 "Le bestiaire dans la publicité", et 14 "Sens dénoté, sens connoté", op. cit., p. 128-129.

21 "Ce travail de relation entre les indices perçus visuellement et les connotations qu'elles entraînent est délicat car dépendant à la fois de la sensibilité de l'indexeur et de la culture de l'entreprise, aussi est-il l'objet de beaucoup de méfiance.", écrit Elisabeth Guilbaud (1995, p. 36).

22 <http://www.sygma.fr>

23 La fonction "Aide" en page d'accueil permet d'obtenir des explications sur chacune des rubriques et sur les procédés de recherche documentaire dans la section "reportages". A noter que certaines photos ne sont pas accessibles en raison d'une "restriction de diffusion".
} 
qui "décrivent" le visible (agents, objets, situations, gestes, composants), et d'autre part, ceux qui impliquent une connaissance des causes et du contexte d'une scène. Ainsi, une date, un lieu, un nom, une fonction parfois (quand un agent n'effectue pas une action directement liée à sa nature ou ses activités) ne sont connaissables que grâce à des sources extérieures à l'image, émanant très souvent du photographe lui-même. Si une scène de réunion était détachée de toute information préalable ou annexe, elle ne représenterait qu'une scène... de réunion ! Pourrait-on approximativement la situer dans le temps et l'espace en fonction des particularités du lieu (décor, appareils, etc.), des traits physiques, vestimentaires ou comportementaux, et de certaines caractéristiques techniques du support (couleur, objectifs particuliers), qu'elle resterait néanmoins inutilisable en tant que document d'actualité.

Le travail d'indexation dans ce domaine repose non seulement sur l'observation analytique mais aussi sur le renseignement. Après avoir relevé les critères déterminants des notices (personnes, objets, actions, situations, etc.), des tests d'indexation successifs sur certaines photos, comparés ensuite avec les notices effectives, mettront en valeur le fait qu'une photographie d'actualité, même si ce n'est pas toujours vérifiable ensuite dans la presse, demande une légende et une notice circonstanciées dès la source. On percevra concrètement, de cette façon, que son utilisation journalistique, en tant que preuve ou témoignage, n'est possible qu'à condition d'être correctement référencée, en l'occurrence documentée.

\subsubsection{Muséographie : Joconde et Vidéomuseum}

Destinées à la mise en mémoire et à disposition de reproductions et données textuelles concernant des oeuvres qui appartiennent aux collections publiques françaises, Joconde et Vidéomuseum ${ }^{24}$ offrent des matériaux de prédilection pour comprendre les questions particulières que posent l'image artistique dans le cadre d'une indexation par sujet. A la consultation des photographies en tant que documents iconographiques, on ajoutera l'étude des critères retenus pour renseigner les divers champs des notices d'accompagnement.

Comparativement aux autres, ces banques entourent d'un soin plus marqué la collecte d'information pour situer les oeuvres dans le temps et l'espace (auteur, origine géographique, date, période, lien avec des sources littéraires, historique, localisation actuelle, etc.), de même qu'elles apportent plus de précisions sur leurs techniques d'exécution ou leur matérialité. Ces indications muséographiques donnent les renseignements nécessaires à la bonne compréhension des caractères particuliers de chaque pièce et de son contexte de création, garantissant une interprétation correcte. Autant de données utiles pour reconstruire ce que Laurent Gervereau (1994, p. 54-63) nommerait le "contexte en

\footnotetext{
24 Précisons que le site Joconde <http://www.culture.fr/documentation/joconde> ne comporte pas encore toutes les illustrations attachées aux notices et que le site Vidéomuseum <http://www.videomuseum.fr $>$ ne présente qu'une version expérimentale de sa banque, mais, en l'état, ces deux sites sont parfaitement utilisables dans un cadre pédagogique.
} 
amont" dans une analyse d'image, mais qui répondent aussi, spécifiquement, aux clés d'accès multiples pour une prospection documentaire. Si un historien souhaite comparer un ensemble de tableaux du $17 \mathrm{e}$ siècle des écoles française et flamande, il possèdera avec ces premiers critères (siècle, objet, école) de quoi interroger la base Joconde, et déjà un embryon d'exercice méthodologique dans la recherche de sources iconographiques. Si un professeur d'arts plastiques est intéressé par l'utilisation du tissu dans l'art contemporain, il obtiendra quelques réponses avec Vidéomuseum. En modifiant la nature des demandes par la variation des critères et des termes, il est possible de reconstituer peu à peu sinon le bordereau de saisie - ce qui supposerait un travail très approfondi - du moins son schéma principal.

On complètera l'observation de ces critères, relevant globalement de la catalographie ${ }^{25}$, par ceux qui dépendent plus exactement de l'indexation par sujet. Le problème, à nouveau posé, des moyens de formuler de la manière la plus pertinente ce qui décrit une image reçoit deux réponses divergentes. Vidéomuseum ne prévoit pas d'indexation et, par conséquent, d'interrogation par sujet, tandis que Joconde a mis en place une indexation de la représentation dont la méthode, mise au point par François Garnier, est expliquée dans son ouvrage Thesaurus iconographique (1984). Ici, des réflexions s'imposent qui pourront alimenter des axes de travail sur les potentialités et les limites de chacun des systèmes (Régimbeau, 1998).

Avec Vidéomuseum, on réfléchira sur les accès qui, en l'absence d'un champ "sujet", sont susceptibles d'orienter malgré tout vers les contenus iconiques et sémantiques d'une oeuvre d'art. Le titre et les matériaux fourniront de ce point de vue des indicateurs plus ou moins fiables mais néanmoins intéressants à analyser dans le cadre de l'esthétique contemporaine. On notera simplement qu'une étude de la fonction du titre dans ses rapports avec l'oeuvre pourrait offrir un support des plus stimulants à un cours de littérature sur le même sujet.

Avec Joconde, une fois compris les principes d'interrogation par sujet qui, entre autres, introduisent aux genres définis par l'histoire de l'art (ex : portrait, scène mythologique, nature morte, paysage, etc), on abordera des exercices d'application en examinant les différentes parties d'une "phrase" d'indexation descriptive. S'il convient d'apprendre à formuler dans la position de l'utilisateur, on comprend d'autant mieux la formulation en prenant la position de l'indexeur ${ }^{26}$. Dans cette optique, en graduant la complexité - car distinguer le genre, puis nommer les agents, les fonctions, les valeurs de plan, les caractères physiques ou matériels, les actions, les attributs, les lieux, le décor, etc. ne va pas de soi - on établira des tests sur certaines images repérées au préalable pour cerner la part interrogeable d'une représentation. Du principal au secondaire, de l'essentiel à l'accessoire, des fausses évidences de représentations extrêmement codées au danger d'inventer des codes où il n'y en a pas, on

\footnotetext{
25 Cf. les normes de catalogage de l'image fixe, AFNOR, FD Z 44-077, sept. 1997, dans Formation des bibliothécaires et documentalistes, AFNOR, 1998.

26 Cf. dans ce numéro "Décris-moi une image" de Marie Després-Lonnet sur les rapprochements à concevoir entre indexeurs et utilisateurs pour affiner les registres d'accès.
} 
dégagera en même temps une matière à discussion qui alimentera, sans s'y substituer, toute pédagogie de déchiffrage d'un document d'histoire.

\subsubsection{Recherche de l'image par l'image : Surfimage}

$\mathrm{Au}$ vu des difficultés que suppose la recherche d'images par l'entremise des mots, des systèmes documentaires qui s'appuient prioritairement sur l'interface de l'image elle-même ont été mis au point, depuis des années ${ }^{27}$. Surfimage est un de ces projets ${ }^{28}$. A partir d'une image-type choisie dans un lot, on obtient des affichages mosaïques où chaque photographie présente une variante proche de l'image sélectionnée. C'est un peu comme si l'on faisait défiler les planches contacts d'une séance de studio ou d'un reportage en proposant un choix extrêmement fin entre deux clichés. Telle interrogation sur le "visage" permettra, par exemple, de choisir entre deux directions de regard pour un même personnage. Une autre interrogation sur des "gélules" affichera les gros plans de configurations diverses où l'oeil pourra préférer un ensemble compact à un autre plus aéré. Les critères ne passent donc plus par des mots d'accès, seraient-ils extrêmement précis, mais par le balayage visuel des mosaïques et la sélection progressive des caractéristiques souhaitées.

Si le principe a de quoi séduire, il faut aussi comprendre qu'il n'est opératoire que dans le cas de collections homogènes, composant des subdivisions de "photos-références", où les variations infimes de détails supposent des recherches guidées par les ressemblances plutôt que par les différences. Un tel système d'interrogation inclus dans une agence d'illustration généraliste ou dans une agence de photojournalisme n'aurait son utilité que dans le deuxième temps d'une requête, quand on souhaiterait mieux affiner la réponse à partir d'un lot d'images proches par leur sujet et leurs composants. Mais on serait vite limités pour accéder à l'extrême variété des domaines et des stocks par la seule interface des images ; à moins d'en afficher des mosaïques infinies qui feraient perdre alors un temps considérable, bien plus qu'avec les critères traditionnels des mots.

On pourra, cependant, à travers ce site, mesurer les progrès réalisés dans ces travaux sur la recherche de l'image "par l'image", dite aussi (de manière assez ambiguë) méthode "d'indexation par le contenu", et s'interroger sur les critères qui déterminent les séries et les "enchaînements" de photographies à l'intérieur des séries. Chaque lot met en oeuvre des segmentations particulières. Si la collection de portraits aux expressions diverses semble extraite de fichiers anthropométriques, une série de photographies sur un lieu urbain mettant en scène une voiture répond, quant à elle, à un découpage cinématographique qui donnerait les photogrammes significatifs de chaque plan.

Quand une banque comme Surfimage sera couplée à un système de reconnaissance graphique, elle saura satisfaire des demandes qui

27 Cf. en particulier les travaux d'Henri Hudrisier.

28 <http://www-rocq.inria.fr/imedia>. Présentation du projet par Chahab Nastar (1999). 
traduisent par un dessin schématique, un "rough", le type d'image souhaité. On sera parvenu, à ce stade, à l'interrogation iconique d'une indexation iconique. Déjà, certains laboratoires proposent des solutions (Mandard, 2000). Sera-t-on pour autant dispensé d'annexer des mots aux images ? Intervient ici la nécessité de séparer aspects iconiques et sémantiques. Si l'on parvient à regrouper, de manière mathématique, des degrès de similarité, basés sur la structure ou les densités par rapport à une image-type, qui permet, par exemple, à un logiciel de reconnaître telle structure iconique par rapport à une autre, pourra-t-on retrouver une image évoquant la solitude en passant de la photographie d'un S.D.F. prise dans une rue à celle d'un arbre isolé dans une plaine ? Sans organiser des ensembles reliés par le sens (champs sémantiques ou catégories) et non plus par leur structure iconique, on sera dans l'incapacité d'obtenir ce genre de relation. Outre l'intérêt pédagogique d'illustrer ce que recouvre l'expression "recherche de l'image par l'image", quelque peu mystérieuse, ce site offre un sujet de réflexion sur l'efficacité relative des clés d'accès, qu'elles soient verbales ou visuelles.

\subsubsection{Images scientifiques et techniques : Institut National de Recherche en Informatique et en Automatique}

En ce qui concerne l'image et la photographie appliquées à la pratique et aux problématiques des sciences de la matière et de la vie, le site de l'INRIA présente non seulement une photothèque mais, comme nous l'avons mentionné, propose aussi une liste de liens à travers sa "Webthèque" qui constituent d'autres pistes de découverte pour des domaines tels que la physique, l'imagerie médicale ou les Systèmes d'Information Géographique (S.I.G.). Pour s'initier, cependant, aux procédés d'interrogation documentaire d'un fonds lié aux recherches et activités d'un laboratoire, ce site sera un point de départ des plus intéressants puisqu'il permet d'observer en même temps la question des critères descriptifs appliqués à l'image scientifique et les possibilités d'accès aux images.

La page d'accueil de la rubrique "Photothèque" nous introduit d'emblée dans la souplesse d'un système qui prévoit trois accès : 1) par l'image, grâce à une mosaïque aléatoire regroupant une sélection de photographies de personnes, de lieux, de matériels et de schémas accompagnées de leur légende ; 2) par des termes pré-définis se rapportant à des divisions thématiques (ex. : Algorithmique, Automatique, Calcul scientifique, Interaction homme-machine, etc), ou, enfin, 3) par la recherche en texte intégral sur tous les champs ; les mots pouvant se formuler avec une troncature et se combiner avec les opérateurs booléens (et, ou, non). Du point de vue ergonomique, on constatera la clarté et la simplicité d'une mise en page qui renforcent l'efficacité de l'interrogation en n'alourdissant pas outre-mesure les données à transférer et place le consultant devant les choix principaux.

En partant des divisions thématiques, on obtiendra des images qui résument les axes de recherche, fondamentale ou appliquée, de ce 
laboratoire, orientés vers le calcul, l'informatique, la robotique et l'automatique dans divers secteurs (industrie, santé, communication, etc.). Ce premier contact, complété par la lecture des notices de quelques images, donnera, de plus, l'occasion d'approcher la spécifité des lexiques rapportés aux sujets représentés. Une fois compris la nature du fonds et ses utilisations potentielles, les choix multicritères pourront ensuite s'exercer sur l'ensemble des informations textuelles réunies et organisées autour des images grâce à l'interrogation en texte intégral : la légende, le nom des projets de recherche, la description de contenu en langage naturel et les descripteurs en langage contrôlé seront ainsi sollicités.

On remarquera que l'indexation connotative n'intervient pas ici comme un critère d'accès. On pourrait l'imaginer cependant avec certaines photographies prévues pour la communication auprès des médias ou en modifiant, simulant, l'indexation d'un sujet hors de son cadre scientifique d'origine. Les notations de contenu, dans des titres strictement dénotatifs ; des légendes, qui atteignent parfois la taille d'un petit paragraphe ; et des descripteurs, dont l'activation permet par ailleurs d'accéder à d'autres images associées, servent avant tout à cerner les caractéristiques précises du sujet représenté à l'aide d'un vocabulaire très spécialisé. Mais, la spécialisation des notions, des phénomènes et des techniques décrits dans cette banque d'images ne fera pas oublier la grande facilité de sa consultation. Car en navigant du texte à l'image et réciproquement, ou de lien en lien à travers la fonction hypertexte ; en vérifiant ce qu'on obtient par le jeu des requêtes thématiques et en texte intégral, on comprendra qu'on a affaire ici à l'architecture d'un système où la complémentarité entre les mots et les images est non seulement manifeste mais encore opératoire. C'est cet aspect que l'élève pourra étudier, puis comparer à d'autres solutions rencontrées.

\section{PERSPECTIVES PÉDAGOGIQUES}

\subsection{Des paliers méthodologiques}

Si l'on observe les différentes approches préconisées en matière de pédagogie de l'image, on retrouve ce que Christian Metz (1970, p. 164-165) séparait en deux apprentissages : " $1^{\circ}$ reconnaître un certain nombre de configurations signifiantes spécifiquement iconiques [...]", recouvrant "l'initiation à la rhétorique de l'image" ; "2॰ reconnaître un certain nombre de symboles largement culturels", [...] autrement dit : "des symboles sociaux plus ou moins extra-iconiques dans leur nature mais fréquemment mis en images".

Cette division méthodologique n'est pas sans rappeler la progression suivie dans Petite fabrique de l'image où chaque chapitre envisage deux étapes destinées, pour la première, à reconnaître une 
fonction rhétorique puis, une seconde, à la connaissance de cette fonction dans des systèmes sémiotiques et des cadres culturels particuliers ; ainsi des codes de la perspective et de la profondeur abordés à partir d'un trompe-l'oeil urbain puis analysés dans les représentations de l'art préhistorique, de la peinture et enfin de la photographie. On reconnaît également, sous des intitulés et des subdivisions différentes, ce souci d'articuler aspects spécifiquement iconiques et élargissement sémantique dans le traité didactique d'Yves Berger (1968), Découverte de la peinture, comme dans le guide plus récent de Laurent Gervereau (1994) sur l'analyse des images. Dans tous les cas, il s'agit de structurer l'apprentissage sur un mode progressif et cumulatif partant de l'inventaire des éléments en jeu pour aboutir à leur interprétation. Que celle-ci soit destinée, comme le propose Christian Metz (1970, p. 166) dans une perspective sémiologique à "desserer autour de [l'enfant] l'emprise des idéologies et des rhétoriques régnantes" ou, dans une perspective antitraumatique, à "contribuer à la mise en place d'une culture du doute généralisé vis-à-vis de toutes les images [...]" comme le préconise Serge Tisseron (2000, p. 135) - opposé, d'ailleurs, à la prééminence de l'approche sémiologique - la visée d'une éducation à l'image est celle d'une familiarisation avec l'examen critique de ses propriétés et le décodage sémantique de ses contenus culturels.

De tels objectifs supposent un travail de défrichage où les techniques documentaires sont susceptibles de jouer un rôle d'aiguilleur important puisqu'elles nécessitent, comme dans toute analyse, un va et vient constant entre la reconnaissance des entités constitutives de l'image et l'interprétation du sens de leurs associations, complétée ou non par la connotation.

Dans un langage pré-défini ou avec les mots du langage naturel, cependant régi par des nécessités documentaires, l'impératif du traitement d'un document est d'aboutir à une représentation des contenus iconiques et sémantiques, à savoir ce qui est montré et ce dont il s'agit ; regroupant ce que pour le texte on nommerait occurrences lexicales et sémantiques. C'est ce dernier aspect qui pose les problèmes les plus difficiles à résoudre dans l'indexation automatique : comment condenser du sens dans une information secondaire avec des termes ou des images qui représentent un contenu primaire sans que ces termes, dans le cas du texte, ou ces traits iconiques, dans le cas de l'image, soit effectivement présents dans ce contenu primaire.

Généralement - car selon le contexte d'application, les variations sont assez amples - on distingue en documentation les niveaux catalographique, descriptif et connotatif, ces deux derniers pouvant être réunis dans un niveau sémantique.

La rédaction de la notice catalographique assigne à l'image, dans ses données minimales : un titre, un auteur, une date, un éditeur, un support et une technique.

La détermination des sujets inaugure l'indexation proprement dite. Sujets principaux et secondaires relevés à partir de l'observation, des indications fournies par l'auteur ou par des sources d'informations 
annexes (exemples : tel nom de personnalité, tel évènement, tel lieu), ainsi que l'identification d'éléments situant la scène et l'action dressent une synthèse descriptive ou dénotative selon les critères de Quintilien (Où, Quand, Qui, Quoi, Comment ?) qui extrait les caractéristiques dominantes de l'image (Kattnig et Léveillé, 1989, p. 62-63). Quant au niveau connotatif, moins utilisé que les autres, il fait appel à la symbolique ainsi qu'aux associations d'idées plus ou moins explicites impliquées par l'image. Or ces distinctions de niveaux sont celles que l'on retrouve dans les grilles et méthodes d'analyse sémantique de l'image dont le schéma comprend trois parties ${ }^{29}: 1$ ) l'identification du document ; 2) la description (information à déchiffrer) ; 3) l'interprétation (information à décrypter).

On peut donc concevoir des passages entre analyse de contenu et analyse documentaire. La tâche du pédagogue sera d'expliquer les différences de perspective et de résultat. Car si l'on attend un commentaire composé d'une analyse de type historique ou littéraire, il n'en va pas de même dans l'optique d'une analyse documentaire où le "commentaire" se solde par l'énumération, la synthèse et la concision, en un mot la condensation. Qui plus est, cette opération est canalisée par des normes, des bordereaux pré-établis, des termes pré-organisés qui exigent de distinguer entre un niveau de réception global, ouvert à de multiples interprétations et une traduction orientée qui tient compte d'un nombre limité de choix. Néanmoins, en comparant les deux méthodes, on rencontrera très vite des points de contact qui faciliteront leurs compréhensions respectives.

\subsection{Décrire et dénombrer : apprendre à voir pour transmettre}

Il suffit d'observer le comportement d'élèves durant des exercices d'indexation pour comprendre que, d'une part, le travail d'explicitation des contenus ne va pas sans lutte, dans de nombreux cas, contre la "loi" de l'évidence, et que, d'autre part, l'attention est souvent happée par le caractère expressif de l'image.

Mettre des mots sur ce qui semble visuellement évident, en particulier pour l'image figurative, suppose de dépasser le refus, qui mêle gêne et peur du ridicule, de nommer "ce qui va de soi". L'élève pense qu'il est inutile de désigner ce que tout le monde est en mesure de voir : "pourquoi répéter ?". Or si l'on se place d'un point de vue documentaire en spécifiant l'intérêt de dénombrer les sujets pour les rendre accessibles, on va contre ce premier mouvement de retenue en donnant, en quelque sorte, une légitimation à l'inventaire descriptif. Nombreux sont les problèmes qui interviennent après l'ouverture de ce verrou mais ce coefficient de motivation ajouté à la description se révèle souvent efficace pour désamorcer un repliement sur l'implicite. Il rejoint par là les processus de verbalisation dont on connaît l'importance psychologique. En déplaçant l'acte de décrire, commandé d'habitude par

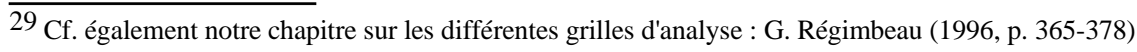


la réception individuelle, vers la transmission, qui implique une dimension collective, on peut s'attarder sur l'importance des informations à relever avant de les proposer à la reconnaisance des autres : il ne s'agit plus seulement de voir mais encore de voir pour transmettre.

Les composantes formelles et esthétiques de l'image déclenchent des jugements de goût beaucoup plus intenses que celles du texte. Même en présence d'une image qui ne cherche pas à émouvoir : photographie frontale en plan d'ensemble sans recherche particulière d'une scène urbaine ordinaire, par exemple, c'est aussitôt son atmosphère qui appelle des réactions affectives et subjectives : le calme ou l'agitation, la laideur ou la beauté supposées, la ressemblance ou la différence avec d'autres situations connues, etc. viennent "au devant de la scène". L'image n'est plus simplement la trace d'une réalité extérieure (studium), elle entre dans ma réalité (punctum) ${ }^{30}$. Lorsqu'il faut revenir sur le dénombrement des éléments en présence, les difficultés commencent : s'agit-il d'une route ou d'une rue ? doit-on parler de trafic ou de circulation ? au loin, voit-on une tour ou un immeuble ?, etc. Organiser un relevé en fonction de rubriques pré-déterminées, comme on en trouve dans n'importe quel bordereau d'indexation, c'est déjà comprendre qu'au delà de l'immersion visuelle première, il est possible de définir des points de vue dans l'image et sur l'image, et ce à plusieurs niveaux.

\subsection{Des images et/ou des mots}

A travers les divers systèmes, on a pu observer que la catégorisation d'un élément visuel obéit à plusieurs facteurs qui dépendent principalement, d'une part, de la nature des images et, d'autre part, des moyens et des objectifs de la banque d'images. Une photothèque d'illustration n'utilisera pas les mêmes critères qu'une banque de données sur les oeuvres d'art.

Les progrès informatiques permettent de prendre en compte maintenant dans les moyens mis à la disposition de l'indexeur et de l'usager l'interface de l'affichage mosaïque qui a des conséquences dans l'opération indexatoire. La possibilité de visualiser autorise l'utilisateur à effectuer des va-et-vient permanents entre le verbal et le visuel. On retrouve ce que Henri Hudrisier avait étudié (1983, p. 143) : "La voie linguistique de la documentation image n'est donc pas la seule voie possible. Associée aux 'vieilles recettes' de la documentation (les mots clés), l'organisation des photographies par paquets, par 'nuages' [...] peut être une voie rapide pour retrouver des photographies dans un stock". En accordant la primauté à la vision directe d'ensembles préorganisés par thèmes, ou parfois aléatoires, le consultant peut accéder aux documents qui correspondent le mieux à ses attentes (image mentale). Mais ces recherches ne s'effectuent pas sans recours à une organisation préliminaire. Même dans le cas des recherches d'images "par l'image", il y a necessité d'un classement qui organise un ensemble en collection. Quoi qu'il en soit, les pratiques actuelles d'analyse et

30 Selon la distinction de Roland Barthes (1980, p. 48-49). 
d'indexation, en fonction des unités documentaires de la pièce ou du lot, restent encore dominées par la nécessité de chercher des correspondances entre les mots et les images, même si cette contrainte est relativisée.

En reprenant ces logiques d'indexation, le repérage de l'importance accordée aux sujets dominants et secondaires peut donner lieu à des exercices sur la profondeur de l'indexation entendue selon deux axes : qualitatif et quantitatif.

L'axe qualitatif concerne la recherche de faisceaux de termes définissant au mieux un sujet. Par exemple : "forêt", mais encore "feuillu", mais encore "chêne", mais encore "été", mais encore "France", mais encore : "Périgord", etc. ; puis de précisions d'ordre morphologique pouvant inclure la lumière, la couleur, l'optique, la valeur de plan, etc. selon les critères définis par Ginette Blery ${ }^{31}$; et enfin la recherche de termes connotatifs, telle qu'on la pratique dans les agences d'illustration. Ces diverses opérations dépendant des éléments identifiables directement dans l'image et d'informations annexes disponibles.

L'axe quantitatif correspond, quant à lui, à une recherche d'exhaustivité dans la caractérisation des composants iconiques : à propos du sujet principal mais aussi des éléments secondaires et des détails. Ainsi, on pourra affecter des termes aux divers composants intervenant dans les différentes parties de l'image. Organiser une liste de "candidats" descripteurs en tenant compte de critères tels que les plans (du premier à l'arrière plan en passant par le plan intermédiaire) ou le critère du sujet (principal et secondaire) permet d'abord de tester concrètement des notions et ensuite d'éprouver une teminologie souvent flottante. Quelles différences établir, par exemple, entre thème, sujet, détail et motif ? Quels éléments, une fois repérés dans l'image, peuvent répondre à cette hiérarchie ?

En multipliant les exercices de ce type, qui devront sans cesse être replacés dans le cadre de ressources adaptées à un contexte particulier, l'élève entre progressivement dans un domaine qu'il aborde ordinairement à travers le prisme du commentaire. L'indexation joue ici le rôle de palier dans l'acquisition des connaissances. Le dénombrement des composants invite à fixer l'attention sur la matière à décrire quand souvent, la mise en texte, incline l'élève à focaliser son esprit sur la manière de dire. La récapitulation, la liste, la répartition des termes offrent un moyen de diversifier les chemins d'accès à la description en réévaluant les fonctions du relevé par rapport à la narration.

\section{Conclusion}

Le domaine des photothèques et des banques d'images constitue par lui-même un complément utile aux connaissances générales. Le stockage et la propagation des images correspondent à des fonctions et des nécessités éducatives, culturelles et économiques ; on le conçoit pour l'écrit mais on oublie souvent de l'envisager pour l'image. Évoquer ses

\footnotetext{
${ }^{31}$ Rappelés par C. Kattnig et J. Léveillé, op. cit., p. 49-59.
} 
sources, c'est éclairer d'une autre manière ses manifestations médiatiques et éditoriales. En précisant l'origine des sites et leur destination, on aborde, en outre, un aspect des réseaux qui relève de la "cartographie". Même s'il est illusoire de croire qu'on puisse dresser une carte exhaustive d'Internet, il convient de garder à l'esprit les correspondances qu'il entretient avec l'organisation économique, politique et sociale (les réseaux sous les réseaux) sous peine d'arrêter artificiellement son approche à des dimensions techniques.

La banque d'images est un outil de connaissance. Les collections d'images relatives à l'histoire, aux sciences ou aux oeuvres sont des aides à l'apprentissage pour l'étude d'un phénomène, d'une période ou d'un art. Il faut cependant qu'elles comportent un appareillage suffisant pour être exploitées comme matériau (document historique, témoignage ou démonstration), notamment des notices et légendes claires et complètes. On pourra, sur ce point, procéder à des vérifications, qui serviront à cerner des impératifs documentaires mais aussi à comprendre que l'emploi du document figuré obéit à certaines conditions amplifiant, réduisant ou distordant l'interprétation (les dangers de l'anachronisme par exemple).

En découvrant les diverses particularités des répertoires d'images et leur inscription dans des réseaux, on perçoit plus facilement, à travers leurs présentations, leurs rubriques, leurs accompagnements textuels avant cette espèce de valeur objective et autonome procurée par l'édition - qu'ils suivent essentiellement les tendances et les besoins de la culture visuelle d'un moment. En étudiant les enjeux documentaires des ressources d'Internet, l'élève, sera donc confronté aux règles et aux usages qui déterminent la place et le sens d'une image dans un stock avant qu'elle ne rejoigne le flot des images éditées ; découvrant, par là, la relativité de ses utilisations. Il pourra, de même, en observant les critères d'indexation, comprendre quels aspects de l'image sont retenus et ceux qui resteront inaccessibles à l'interrogation ; autre forme de leçon pratique pour identifier ce lien paradoxal, parfois étroit, parfois distant, qui unit le texte et l'image.

On aura remarqué, à la lecture de cet article, que pour chacun des sites mentionnés, certains axes ont été privilégiés au détriment d'autres qui peuvent s'avérer tout aussi fructueux ; ces choix, se réfèrant en grande partie à une expérience d'enseignement en I.U.T. où il faut combiner culture de l'image et apprentissage documentaire, sont donc relatifs. Il demeure qu'à travers ce court panorama, on saisira peut-être qu'un des "supports" actuels de la pédagogie de l'image consiste en des approches transversales et concrètes qui faciliteront non seulement la connaissance mais encore l'appropriation critique des TICE. Un domaine et un projet où les spécialistes du traitement et de la formation en matière d'information, notamment les professeurs documentalistes, ont, nous semble-t-il, un rôle important à jouer.

Gérard Régimbeau. Sept. 2000. 
Maître de conférences de Sciences de l'information et de la communication. Chercheur au LERASS (Laboratoire d'Études et de Recherches Appliquées en Sciences Sociales). Equipe MICS (Médiations en information et communication spécialisées). Toulouse 3. Merci à Arlette Bouzon, Viviane Couzinet et Francine Pavan pour leur relecture.

\section{RÉFÉRENCES}

BARTHES Roland (1964). Rhétorique de l'image. Communications, 1964, n 4 , p. 40-51.

BARTHES Roland (1980). La chambre claire : note sur la photographie. Cahiers du cinéma : Gallimard : Le Seuil. 192 p. ISBN 207-020541-X.

BERGER Yves (1969). Découverte de la peinture. Gérard et $C^{\circ} .3$ vol. (267 p., 248 p., 253 p.). Marabout université ; 171-173.

COURBIÈRES Caroline (2000). De la mode et des discours au regard de l'indexation documentaire. 314 p. Thèse de doctorat de Sciences de l'information et de la communication. LERASS. Université de ToulouseLe-Mirail.

DAUTUN Jean-Pierre (1995). Dix modèles d'analyse d'image. Marabout. 307 p. Parascolaire. Culture générale ; 8032. ISBN 2-50102096-0.

Documents iconiques. Documentation et information. Vocabulaire. Partie 3. ISO 5127-3 / ISO. Première édition 1988. Diff. AFNOR. 17 p. Français-Anglais.

FOZZA Jean-Claude, GARAT Anne-Marie et PARFAIT Françoise (1992). Petite fabrique de l'image : parcours théorique et thématique, 180 exercices. Magnard. 254 p. ISBN 2-210422-90-6.

GARNIER François (1984). Thésaurus iconographique : système descriptif des représentations. Le Léopard d'or. 239 p. ISBN 2-86377032-2.

GERVEREAU Laurent (1994). Voir, comprendre, analyser les images. La Découverte. 191 p. Guides Repères. ISBN 2-7071-2318-8. 
GERVEREAU Laurent, dir. (1999). Peut-on apprendre à voir ? L'image : Ecole nationale supérieure des Beaux-Arts. 380 p. ISBN 284056-076-3.

GUILBAUD Elizabeth (1995). Comment indexer l'image fixe ? Archimag, 1995, n86, p. 33-36.

HUDRISIER Henri (1983). L'iconothèque : documentation audiovisuelle et banques d'images. La Documentation française. 269 p. Audiovisuel et Communication. ISBN 2-11-000998-5.

KATTNIG Cécile et LÉVEILLÉ Janny (1989). Une photothèque : mode d'emploi. Les Éditions d'organisation. 239 p. ISBN 2-7081-1082-9.

LEGUÉRÉ J.P. (1979-1980). La documentation iconographique. BàT, 1979-1980, $\mathrm{n}^{\circ} 19-21$, article en 3 parties.

MANDARD Stéphane (2000). Les moteurs de demain. Le Monde interactif, mercredi 26 janvier 2000, p. III.

METZ Christian (1970). Images et pédagogie. Communications, 1970, $\mathrm{n}^{\circ} 15, \mathrm{p} .164-165$.

MEYRIAT Jean (1993). Documents photographiques et électroniques. In Les sciences de l'écrit : encyclopédie internationale de bibliologie. Retz. Encyclopédies du savoir moderne. P. 152-154.

MOULIS Anne-Marie (1999). La recherche d'images sur Internet. Documentaliste-Sciences de l'information, 1999, vol. 36, n³, p. 179185.

NASTAR Chahab (1999). Indexation et recherche d'images : enjeux, méthodes et perspectives. IDT 99 [en ligne]. [visité le 10.10.2000]. URL : <http://www-rocq.inria.fr/imedia/publications.html>.

RÉGIMBEAU Gérard (1996). Thématique des oeuvres plastiques contemporaines et indexation documentaire. Presses universitaires du Septentrion, 1998. 2 vol. 657 p. Thèse à la carte. ISBN 2-284-00659-0.

RÉGIMBEAU Gérard (1998). Accès thématiques aux œuvres d'art contemporaines dans les banques de données. Documentaliste-Sciences de l'information, 1998, vol. 35, $\mathrm{n}^{\circ} 1$, p. 15-23.

TISSERON Serge (1996). Le bonheur dans l'image. Synthélabo. 137 p. Les Empêcheurs de penser en rond. ISBN 2-908602-79-2. 
TISSERON Serge (1998). Y a-t-il un pilote dans l'image ? Six propositions pour prévenir les dangers de l'image. Aubier. 176 p. ISBN 2-7007-2400-3. 